\title{
Gestão do sucesso de um projeto de sistemas de informação realizado no setor público - research-in-progress
}

\author{
Nilton Takagi ${ }^{1,2}$, João Varajão ${ }^{3,4}$, Thiago Ventura ${ }^{1}$, Daniel Vecchiato ${ }^{1}$, Raphael \\ Gomes $^{1}$ \\ ${ }^{1}$ Instituto de Computação - Universidade Federal de Mato Grosso (UFMT) \\ Cuiabá - MT - Brasil \\ ${ }^{2}$ PDSTI - Universidade do Minho (UMINHO) \\ Guimarães - Portugal \\ ${ }^{3}$ Departamento de Sistemas da Informação - Universidade do Minho (UMINHO) \\ Guimarães - Portugal \\ ${ }^{4}$ Centro ALGORITMI - Universidade do Minho (UMINHO) \\ Guimarães - Portugal \\ \{nilton, thiago, daniel, raphael\}@ic.ufmt.br, varajaoddsi.uminho.pt
}

\begin{abstract}
Despite the significant developments in project management methodologies and tools, achieving success is still a challenge for managers. In this scenario, the experimentation of a model that incorporates activities to characterize and manage success throughout a government information systems project was initiated. It includes activities to identify success criteria and factors using focus group dynamics and the Success Canvas ${ }^{\circledR}$. For the research, a Design Science Research process was adopted, which is in the evaluation phase. Preliminary results show that success concepts are different for each stakeholder, that Iron Triangle criteria are among the most cited, and stakeholder management is a main success factor.
\end{abstract}

Resumo. Apesar da evolução verificada nos últimos anos no que respeita a metodologias e ferramentas da gestão de projetos, alcançar o sucesso é ainda um desafio para os gestores. Neste contexto, foi iniciada a experimentação de um modelo que incorpora atividades que possibilitam caracterizar e gerir o sucesso ao longo de um projeto. Essa experimentação está a decorrer no âmbito de um projeto de sistemas de informação realizado no setor público. As atividades realizadas, contemplando a identificação de critérios e fatores de sucesso, envolveram dinâmicas de focus group com a utilização do Success Canvas ${ }^{\circledR}$. Para a pesquisa foi adotado um processo de Design Science Research, o qual se encontra na fase de avaliação do modelo. Os resultados preliminares mostram que os conceitos de sucesso são diferentes para cada stakeholder, que os critérios do Iron Triangle estão entre os mais citados, e que a gestão de stakeholders é um dos fatores de sucesso mais referido.

\section{Introdução}

Alcançar o sucesso em projetos é ainda um desafio. Analisando as diversas versões de guias de gestão de projetos, como o PMBOK, metodologias como o PRINCE2 ou metodologias ágeis como o Scrum, verifica-se uma evolução em termos de métodos, técnicas e ferramentas para fornecer apoio aos gestores de projetos. No entanto, muitos projetos ainda excedem o orçamento, registram atrasos ou não atingem seus objetivos. Aspectos como a cultura organizacional, competências da equipe e adoção de melhores 
práticas são influenciadores do sucesso geral de um projeto (Cooke-Davies 2002, Chow e Cao 2008), e os critérios para avaliar o sucesso podem diferir de acordo com o tipo e dimensão do projeto (Sato e Chagas 2014, Turner e Xue 2018). Assim, é necessário estabelecer e colocar em prática atividades focadas na gestão do sucesso, devendo as mesmas promover a definição, em cada projeto, dos critérios e dos fatores de sucesso, bem como o seu planejamento, execução, monitoramento e controle (Varajão 2016, Varajão 2018).

Neste contexto, está a decorrer um projeto de investigação que contempla a operacionalização de um processo de gestão do sucesso no âmbito de um projeto de sistemas de informação, desenvolvido no setor público. O projeto tem previsão de término em 2020 e foram já iniciadas atividades de identificação de critérios e fatores de sucesso, seguindo o processo de Design Science Research de Kuechler e Vaishnavi (2008). O trabalho está em curso, sendo neste artigo apresentados resultados preliminares identificados numa sessão de focus group recorrendo ao Success Canvas ${ }^{\circledR}$ (Varajão 2018).

Este artigo está estruturado da seguinte forma: na segunda seção é realizada uma breve revisão de literatura, focando conceitos fundamentais acerca do sucesso (critérios e fatores) e gestão de projetos; a terceira seção apresenta o método de pesquisa definido para a condução da investigação; na quarta seção encontram-se os resultados preliminares, a conclusão, as limitações, e os trabalhos futuros.

\section{Fundamentação teórica}

A perspectiva do sucesso pode ser distinta para cada stakeholder (Shenhar, Dvir et al. 2001). Em gestão de projetos não se encontra uma definição geral de sucesso na literatura ou na prática, uma vez que cada projeto é único. Alguns autores relacionam o sucesso com o conjunto de critérios do Iron Triangle (tempo, custo e escopo), outros concentramse nos resultados da gestão, outros sugerem diferentes dimensões para gerir o sucesso do projeto (Turner 2014, Serrador, Reich et al. 2018). Apesar de nenhuma definição exata de sucesso ter sido encontrada na literatura, há evidências de que a gestão dos critérios e dos fatores de sucesso pode ajudar a colmatar essa lacuna (Varajão, Magalhães et al. 2018).

Os critérios para avaliar o sucesso podem ser definidos como medidas pelas quais o sucesso ou o fracasso de um projeto serão julgados (de Wit 1988). Há vários trabalhos sobre o tema realizados nos últimos anos (Atkinson 1999, Westerveld 2003, Collins e Baccarini 2004, O'Donnell e Galat 2008, Cserháti e Szabó 2014, Turner 2014, Nguyen, Nguyen et al. 2018, Osei-Kyei e Chan 2018). É comum encontrar "conformidade com o cronograma e orçamento" como exemplos de critérios de sucesso. No entanto, existem outros critérios, como a satisfação da equipe, o cumprimento de metas estratégicas, e cumprimento de normas e legislação, o cumprimento de requisitos e especificações, entre muitos outros (Borges e Carvalho 2015, Adabre e Chan 2019).

No que respeita aos fatores de sucesso, estes podem ser definidos como entradas do sistema de gestão que influenciam direta ou indiretamente o sucesso do projeto (de Wit 1988). Similarmente ao que acontece no caso dos critérios, há vários trabalhos focados nos fatores de sucesso (Pinto e Slevin 1987, Dvir, Lipovetsky et al. 1998, CookeDavies 2002, Westerveld 2003, Chow e Cao 2008, Tabish e Jha 2011, Turner 2014, Ferreira, Varajão et al. 2016, Osei-Kyei, Chan et al. 2017, Rezvani e Khosravi 2018, Zuo, Zhao et al. 2018). Estes podem ser relativos às áreas do conhecimento, às fases do projeto 
e até mesmo aos critérios de sucesso. Exemplos de fatores de sucesso incluem o apoio da gestão de topo, identificação dos benefícios para o negócio, gestão de mudanças organizacionais, planejamento da implementação do projeto, ou atualização frequente do cronograma e do orçamento (Tabish e Jha 2011, Ferreira, Varajão et al. 2016).

Integrando a metodologia utilizada no projeto visado nesta pesquisa, está a ser utilizado o processo de gestão de sucesso de Varajão (2018) conforme a Figura 1. O processo define a gestão do sucesso através de nove atividades, enquadradas nos níveis "projeto", "fases" e "iterações". No modelo há duas atividades de planejamento, uma para definir como será a gestão do sucesso no projeto como um todo, e outra como será a gestão do sucesso em cada fase. Definições dos indicadores de desempenho e de resultado a serem usados, quando será feita a avaliação, quem participará nessa avaliação, e a forma de reportar o sucesso, são exemplos de saídas dessas atividades. $\mathrm{Na}$ visão da fase do projeto, há também a identificação dos fatores de sucesso e a definição dos indicadores de desempenho e de resultado, mas sendo neste caso particulares a cada fase. A recolha de dados e a utilização dos indicadores é realizada nas iterações de avaliação, assim como as ações corretivas e preventivas. A atividade de revisão da gestão do sucesso é responsável por fazer uma reflexão sobre o processo durante a sua execução, momento em que podem surgir novos critérios ou fatores de sucesso, os quais devem ser incorporados nas outras atividades.

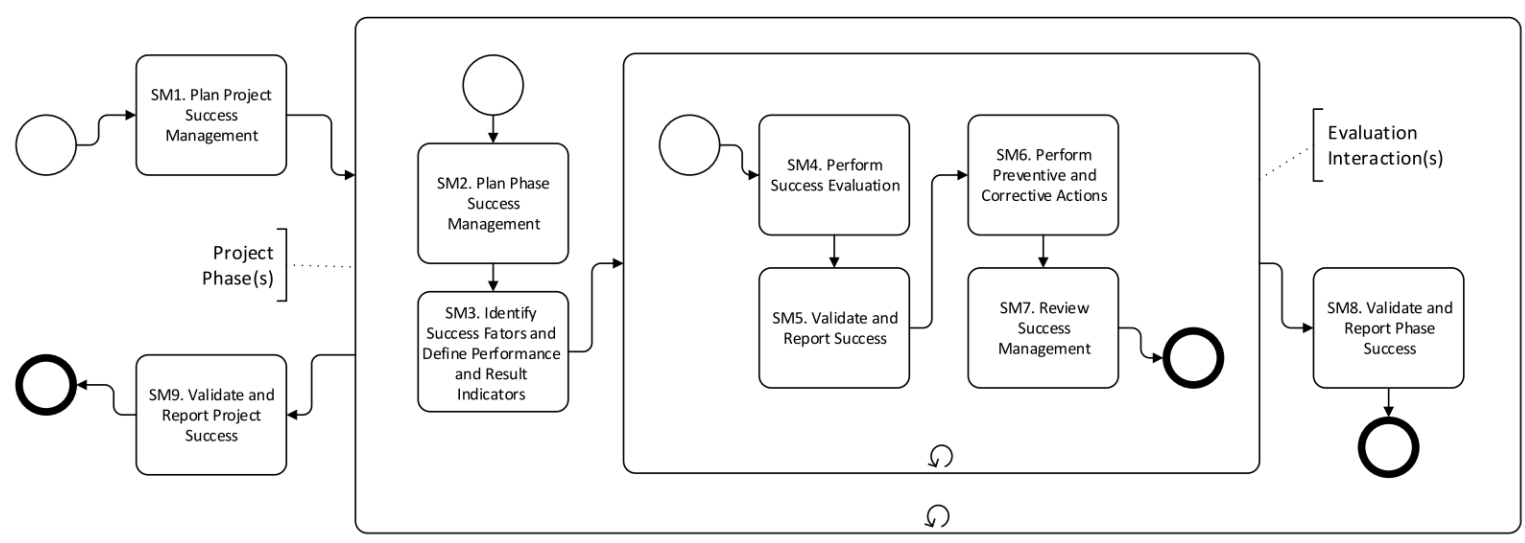

Figura 1. Modelo do processo de gestão de sucesso (Varajão 2018)

O método de investigação definido para atingir os objetivos deste trabalho encontra-se descrito na sequência.

\section{A Metodologia Design Science Research}

A Design Science procura o desenvolvimento de soluções generalizáveis para problemas existentes e o processo deve ser robusto. Deve, também, conter o rigor necessário para que o desenvolvimento e a avaliação sejam realizados com confiabilidade (Hevner, March et al. 2004). Neste trabalho foi adotado o processo de Design Science Research (DSR) de Kuechler e Vaishnavi (2008), conforme apresentado na Figura 2.

Num projeto é necessário identificar os critérios e os fatores do sucesso, e geri-los desde o início até a fase de encerramento, o que não estava a ser realizado no projeto foco deste estudo (Awareness of Problem). Com o objetivo de analisar diferentes possibilidades para a solução do problema identificado, foi realizada uma revisão de 
literatura sobre a gestão do sucesso, tendo sido adotado o processo iterativo proposto por Varajão (2018) (Suggestion).

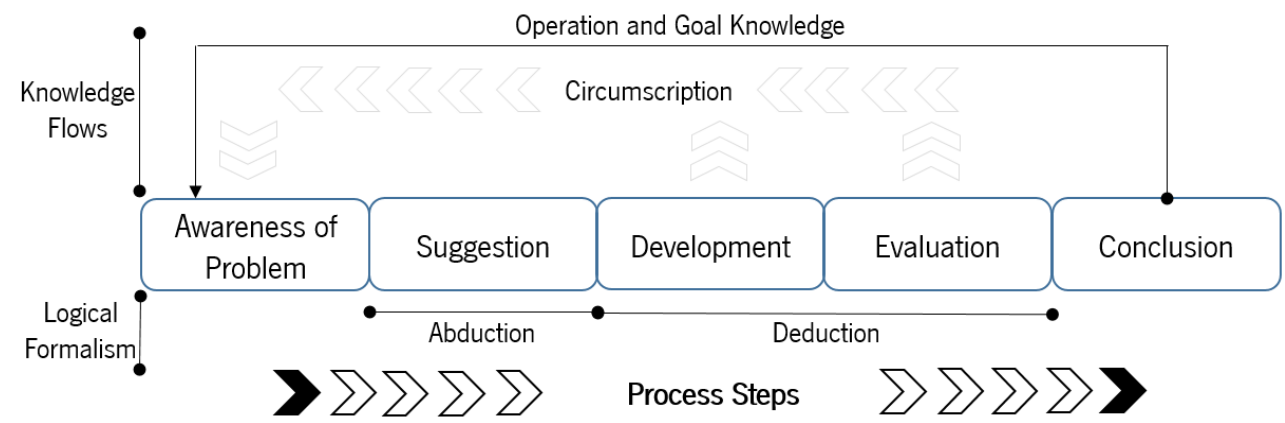

Figura 2. Metodologia de pesquisa, adaptada de Kuechler e Vaishnavi (2008)

A partir do entendimento do problema e da sugestão de solução, foi construído (Development) um modelo integrando a gestão do sucesso na metodologia do projeto. O novo modelo contempla atividades de gestão do sucesso nas fases de planejamento, execução, e encerramento, e nas atividades da monitorização e controle. O processo encontra-se em avaliação (Evaluation), tendo sido iniciado o planejamento e a identificação dos fatores e critérios de sucesso no âmbito de uma sessão piloto de focus group utilizando o Success Canvas $^{\circledR}$ (Varajão 2018). A sessão foi realizada com a participação de líderes da equipe de execução que têm formação acadêmica superior e vasta experiência em projetos similares.

\section{Resultados, análise e conclusão}

Sendo esta uma investigação em curso (research-in-progress), os resultados apresentados nesta seção são preliminares e não refletem todo o processo de gestão do sucesso, concentrando-se apenas nos critérios e nos fatores de sucesso identificados pelos stakeholders.

Em relação aos critérios de sucesso, a área de escopo destacou-se com mais de $45 \%$ dos critérios sinalizados. A referência à finalização e à implantação de todos os módulos previstos foi recorrente entre os participantes. Com pouco mais de $18 \%$ também a área de custos foi das mais referidas, indicando que um critério importante do sucesso é finalizar o projeto dentro do orçamento previsto. Compliance com o cronograma, alinhamento com stakeholders, realização da transferência de tecnologia (knowledge), e melhoria da tomada de decisão, foram outros critérios que emergiram no decorrer da pesquisa. Em relação aos fatores de sucesso, o destaque centrou-se na gestão de stakeholders, com 66,67\%. Diversos stakeholders foram sinalizados como tendo poder para influenciar o projeto e são um aspecto relevante a ter em atenção durante todo o percurso. Surgiram também fatores relacionados com a equipe de execução, gestão do custo e gestão da comunicação. Esta última, relacionando a importância de divulgação do projeto para alcance dos objetivos relativos à utilização dos produtos entregues.

Verificou-se que existe uma clara preocupação com a realização das entregas, estando o escopo em evidência entre os critérios, e a gestão de stakeholders entre os fatores de sucesso. Esses resultados não diferem do que se encontra na literatura (de Wit 1988, Atkinson 1999, Borges e Carvalho 2015). No entanto, é reconhecido que os resultados podem ter um viés, dado que nesta fase a equipa de execução foi a única que participou do processo. Assim, a análise precisa de ser expandida para outros stakeholders 
do projeto, incluindo participantes com poder e interesse do lado do cliente (para além do sponsor). Além da ampliação da participação dos stakeholders para a definição do sucesso, na próxima fase do projeto será iniciado o monitoramento e controle, com vista a verificar se o que foi identificado realmente permanecerá estável com a evolução da maturidade da gestão do sucesso e do projeto.

Com a identificação das áreas de escopo, stakeholders, cronograma e custos, passou a haver uma percepção mais clara dos critérios e fatores de sucesso do projeto relevantes para a sua avaliação. O Success Canvas $^{\circledR}$ (Varajão 2018) revelou-se de grande utilidade para essa identificação. Ter a clarificação das áreas e dos aspetos que requerem mais atenção, auxilia o processo de tomada de decisão, minimizando o desperdício de recursos e o esforço da gestão do projeto.

Como trabalho futuro prevê-se fazer evoluir o processo de gestão do sucesso de modo a facilitar a sua integração com metodologias de gestão de projetos, envolver mais stakeholders-chave do projeto, e iniciar o monitoramento e controle dos critérios e fatores de sucesso.

\section{References}

Adabre, M. A. e A. P. C. Chan (2019). "The ends required to justify the means for sustainable affordable housing: A review on critical success criteria." Sustainable Development. 27(4): 781-794.

Atkinson, R. (1999). "Project management: Cost, time and quality, two best guesses and a phenomenon, its time to accept other success criteria." International Journal of Project Management 17(6): 337-342.

Borges, J. G. e M. M. Carvalho (2015). "Project success criteria: An exploratory study on the influence of the variables project typology type of stakeholder." Producao 25(1): 232-253.

Chow, T. e D. B. Cao (2008). "A survey study of critical success factors in agile software projects." Journal of Systems and Software 81(6): 961-971.

Collins, A. e D. Baccarini (2004). "Project success - A survey." Journal of Construction Research 5(2): 211-231.

Cooke-Davies, T. (2002). "The "real" success factors on projects." International Journal of Project Management 20(3): 185-190.

Cserháti, G. e L. Szabó (2014). "The relationship between success criteria and success factors in organisational event projects." International Journal of Project Management 32(4): 613624.

de Wit, A. (1988). "Measurement of project success." International Journal of Project Management 6(3): 164-170.

Dvir, D., S. Lipovetsky, A. Shenhar e A. Tishler (1998). "In search of project classification: A non-universal approach to project success factors." Research Policy 27(9): 915-935.

Ferreira, B., J. Varajão e A. Cunha (2016). Success factors of CRM project management - A Literature Review. Atas da Conferência da Associação Portuguesa de Sistemas de Informação.

Hevner, A., S. March, J. Park e S. Ram (2004). "Design Science in Information Systems Research." MIS Quarterly 28(1): 75-105.

Kuechler, B. e V. Vaishnavi (2008). "On theory development in design science research: anatomy of a research project." European Journal of Information Systems 17(5): 489-504. 
Nguyen, P. T., T. A. Nguyen, Q. Nguyen, V. D. B. Huynh e K. D. Vo (2018). "Ranking project success criteria in power engineering companies using fuzzy decision-making method." International Journal of Advanced and Applied Sciences 5(8): 91-94.

O'Donnell, T. K. e D. L. Galat (2008). "Evaluating success criteria and project monitoring in river enhancement within an adaptive management framework." Environmental Management 41(1): 90-105.

Osei-Kyei, R. e A. P. C. Chan (2018). "Stakeholders' perspectives on the success criteria for public-private partnership projects." International Journal of Strategic Property Management 22(2): 131-142.

Osei-Kyei, R., A. P. C. Chan e E. E. Ameyaw (2017). "A fuzzy synthetic evaluation analysis of operational management critical success factors for public-private partnership infrastructure projects." Benchmarking 24(7): 2092-2112.

Pinto, J. K. e D. P. Slevin (1987). "Critical factors in successful project implementation." IEEE Transactions on Engineering Management EM-34(1): 22-27.

Rezvani, A. e P. Khosravi (2018). "A comprehensive assessment of project success within various large projects." Journal of Modern Project Management 6(1): 114-122.

Sato, C. E. Y. e M. F. Chagas (2014). "When do megaprojects start and finish? Redefining project lead time for megaproject success." International Journal of Managing Projects in Business 7(4): 624-637.

Serrador, P., B. H. Reich e A. Gemino (2018). "Creating a climate for project success." Journal of Modern Project Management 6(1): 38-47.

Shenhar, A. J., D. Dvir, O. Levy e A. C. Maltz (2001). "Project Success: A Multidimensional Strategic Concept." Long Range Planning 34(6): 699-725.

Tabish, S. Z. S. e K. N. Jha (2011). "Identification and evaluation of success factors for public construction projects." Construction Management and Economics 29(8): 809-823.

Turner, J. (2014). The Handbook of Project-based Management: Leading Strategic Change in Organizations, McGraw-Hill Education.

Turner, J. e Y. Xue (2018). "On the success of megaprojects." International Journal of Managing Projects in Business 11(3): 783-805.

Varajão, J. (2016). "Success Management as a PM Knowledge Area - Work-in-Progress." Procedia Computer Science 100: 1095-1102.

Varajão, J. (2018). "A new process for success management bringing order to a typically adhoc area." Journal of Modern Project Management 5(3): 92-99.

Varajão, J. (2018). Project Success Canvas ${ }^{\circledR}(V 2)$. University of Minho. Portugal.

Varajão, J., L. Magalhães, L. Freitas, P. Ribeiro e J. Ramos (2018). "Implementing Success Management in an IT project." Procedia Computer Science (138): 891-898.

Westerveld, E. (2003). "The Project Excellence Model $\AA$ : Linking success criteria and critical success factors." International Journal of Project Management 21(6): 411-418.

Zuo, J., X. B. Zhao, Q. B. M. Nguyen, T. Ma e S. Gao (2018). "Soft skills of construction project management professionals and project success factors: A structural equation model." Engineering Construction and Architectural Management 25(3): 425-442. 